\title{
U na mirada epistemométrica de la Revista Médica de Chile y su aporte al conocimiento en Medicina
}

\author{
Manuel Krauskopf ${ }^{1 a}$, Erwin Krauskopf ${ }^{1,2 a}$. \\ A scientometric view of \\ Revista Médica de Chile
}

Background: During the last decade Revista Médica de Chile increased its visibility, measured on citations and impact factor. Aim: To perform a scientometric analysis to assess the performance of Revista Médica de Chile. Material and methods: Thomson's-ISI Web of Science and Journal Citation Reports (JCR) were consulted for performance indicators of Revista Médica de Chile and Latin American journals whose subject is General and Internal Medicine. We also report the h-index of the journal, which infers quality linked to the quantity of the output. Results: According to the h-index, Revista Médica de Chile ranks 4 among the 36 journals indexed and published by Argentina, Brazil, Chile and Mexico. The top ten articles published by Revista Médica de Chile and the institutions with the higher contribution to the journal, were identified using citations. In the Latin American region, Brazil relevantly increased its scientific output. However, Argentina, Chile and Mexico maintain a plateau during the last decade. Conclusions: Revista Médica de Chile increased notoriously its performance. Its contribution to the Chilean scientific community dedicated to Medicine appears to be of central value (Rev Méd Chile 2008; 136: 1065-72).

(Key words: Abstracting and indexing as topic; Journalism, medical; Periodicals as topic)

Recibido el 26 de mayo, 2008. Aceptado el 9 de junio, 2008.

${ }^{1}$ Facultad de Ciencias de la Salud, Instituto Milenio de Biología Fundamental y Aplicada, Universidad Andrés Bello y ${ }^{2}$ Fundación Ciencia para la Vida, Santiago, Chile.

aBioquímico, Ph.D.

$\mathrm{S}^{\mathrm{i}}$ hace poco más de diez años era posible btener una suerte de radiografía que revelara a través de indicadores epistemométricos ${ }^{1}$ el cometido de una revista científica de corriente principal, como aconteció con la Revista Médica de Chile al estudiarla en el contexto de conmemorar sus 125 años de existencia ${ }^{2}$, el rápido desarrollo de registros digitalizados y programas

Correspondencia a: Dr. Manuel Krauskopf, Facultad de Ciencias de la Salud, Universidad Andrés Bello, Santiago, Chile. E mail: manuel.krauskopf@laureate-inc.cl computacionales cada vez más complejos permiten ahora cuantificar en forma más precisa el aporte al conocimiento que surge de las publicaciones sometidas a revisión por pares.

La sociedad del conocimiento que sostiene el desarrollo de los países en un mundo global necesita una preocupación responsable del Estado y del sector empresarial por la investigación competitiva internacionalmente, pero al mismo tiempo, exige accountability por los recursos que la financian. En nuestro país, la inversión en Ciencia y Tecnología (C\&T) continúa siendo claramente precaria $(0,7 \% \text { del PIB el año } 2002)^{3} \mathrm{y}$, por el número total de publicaciones citables se ubica 
en el lugar 44 (años 1996-2006) ${ }^{4}$ bastante más abajo que los lugares 18, 28 y 34 de Brasil, México y Argentina, respectivamente. Países más pequeños como Nueva Zelandia (1,16\%), Finlandia $(3,46 \%)$ e Israel $(4,90 \%)$, aventajan a Chile notoriamente en su inversión en C\&T como porcentaje del PGB $(\mathrm{OECD})^{5}$. Es más, mientras durante la última década Brasil, México, Cuba, Colombia y Uruguay crecieron en publicaciones científicas más que el doble respecto al promedio mundial, Chile exhibió un avance relativo de sólo 1,7 veces $^{6}$. Si se cuantifican los aportes a través de los artículos que conciernen a Medicina, Chile se ubica en el mismo período en el lugar 39. Sus documentos citables, de acuerdo a SCImago ${ }^{4}$ - grupo de investigación de las Universidades de Granada, Extremadura, Carlos III (Madrid) y Alcalá de Henares, dedicado al análisis de la información científica y a su difusión- constituyen en Medicina 8,55\% respecto al total publicado por Chile en el período. Brasil mantiene la posición № 18 que alcanza con la totalidad de sus artículos aunque sus publicaciones en Medicina constituyen $4,2 \%$ y Argentina, con 5,75\% respecto al total, se ubica en esta materia en el lugar 30. SCImago registra 1.753 revistas en el área de Medicina y en la categoría Medicina Miscelánea, cubriendo desde lo básico a lo clínico ${ }^{4}$.

La reciente introducción del "índice-h" por Hirsch $(2005)^{7}$ definido como el número de publicaciones que genera un autor que tiene $h$ artículos citados al menos $\mathrm{h}$ veces ha estimulado cientos de estudios para evaluar a investigadores, organizaciones, revistas, países, etc. Aunque es fácil determinar el índice-h en un universo pequeño de artículos, a poco de su propuesta, ha sido incorporado en importantes bases de datos como el Web of Knowledge (Thomson ISI) y Scopus de Elsevier B.V. Este registro, independiente del anterior, sustenta la información contenida en SCImago Journal \& Country Rank. El índice-h que como todos los indicadores tiene limitaciones, supera a otros referentes cuantitativos porque pondera calidad con cantidad de un modo armónico.

En este contexto nos propusimos analizar el protagonismo de la Revista Médica de Chile en la generación de conocimiento médico abordando, al mismo tiempo, el estado actual de la investigación biomédica en el país respecto a lo que acontece en los países latinoamericanos más comprometidos con la investigación ${ }^{6}$. En particular, nos concentramos en las categorías Medicina General e Interna ${ }^{8}$ y Medicina Miscelánea ${ }^{9}$ por ser éstas las categorías en que los bancos de datos clasifican a la Revista Médica de Chile.

\section{MATERIAL Y MÉTODO}

Para obtener los indicadores que se reportan se utilizaron los datos de Thomson-ISI, el Web of Science y el Journal Citation Reports (JCR). En el Web of Science se consideraron todos los tipos de documentos registrados en la Revista Médica de Chile. El JCR incluye sólo artículos, reviews, notas y correcciones. La información que se obtuvo de los índices independientes de SCImago Research Group que se nutre de los registros de Scopus toma en cuenta los documentos citables que provienen de las revistas. Ellos, en este caso, corresponden a artículos y revisiones.

\section{RESULTADOS Y DISCUSIÓN}

Factor de Impacto e Indice-h de la Revista Médica de Chile. Hace 10 años existía una sola base de datos para obtener indicadores directos sobre el devenir de la Revista Médica de Chile: el Journal Citation Reports. Como se observa en la Tabla 1, en la categoría de Medicine, General \& Internal, al

Tabla 1. Indicadores de revistas categorizadas en M edicina, General e Interna de acuerdo al JC R 2006

\begin{tabular}{|llccc|}
\hline & País & $\mathrm{N}^{\circ}$ de artículos & Factor de Impacto & Índice inmediato \\
\hline Rev Méd Chile & Chile & 202 & 0,405 & 0,089 \\
Medicina-Buenos Aires & Argentina & 100 & 0,272 & 0,09 \\
Rev Invest Clin & México & 39 & 0,243 & 0 \\
\hline
\end{tabular}


año 2006 el registro correspondiente no incluye nuevas revistas respecto del año $1995^{2}$, aunque en el ámbito misceláneo de la Medicina Thomson ha incluido en otra base de datos, el Web of Science (ISI), un creciente número de títulos latinoamericanos. Entre ambos años la Revista Médica de Chile se fortaleció de modo significativo ocupando, de acuerdo a su Factor de Impacto, el lugar 84 de los 103 títulos registrados por el JCR 2006 en la categoría examinada. No obstante que en 1995 el mismo índice ubicó a la Revista Médica de Chile en la posión 81 entre 108 títulos y con un Factor de Impacto de 0,176; el registrado en el año 2006 es 2,3 veces superior. La Tabla 1 muestra que el avance de la Revista Médica de Chile, caracterizado por un Factor de Impacto de 0,405, la posiciona como primera de las tres revistas en su categoría en América Latina. El Índice Inmediato, que concierne al número de citas que provoca un artículo el mismo año de su publicación, también se incrementó.

Aunque el registro del ISI aumentó notoriamente, asociando más títulos de países en desarrollo que hace 10 años estaban bastante ausentes, particularmente de América Latina ${ }^{10}$, otros registros creados recientemente proveen información útil a la hora de querer evaluar investigadores, revistas, instituciones. En efecto, SCImago ${ }^{4}$ ha avanzado rápidamente en una base de datos de amplia cobertura incorporando un elevado número de títulos no incluidos en ISI y articulados a SCOPUS $^{11}$ (que indexa sistemáticamente 15.000 revistas que contienen artículos revisados por pares evaluadores y publicados por 4.000 editores internacionales). El Web of Science (ISI), por su parte, incluye el Science Citation Index Expanded, con acceso a 6.650 revistas líderes en el mundo en ciencia y tecnología; al Social Sciences Citation Index con registro bibliográfico de 1.950 revistas y el Arts \& Humanities Citation Index que sistematiza la información que concierne a 1.150 títulos del área en cuestión. Con características claramente distintas, Thomson (ISI) publica anualmente el JCR que concierne al registro de los indicadores que generan 7.500 revistas de relevancia publicadas en más de 60 países.

$\mathrm{Si}$ bien el Factor de Impacto y el Índice Inmediato que calcula el JCR para distinguir el impacto de las revistas han sido los principales elementos utilizados para caracterizar las publica- ciones de corriente principal, la aplicación del índice-h para perfeccionar este objetivo se extendió rápidamente. Ello aconteció a la par del desarrollo de nuevos y más amplios registros que incorporaron este indicador que armoniza la calidad considerando la distribución de citas de un universo de publicaciones en un periodo determinado. La Tabla 2 señala las 36 revistas que registra SCImago en el área de Medicina, Categoría Medicina Miscelánea, pertenecientes a Argentina, Brasil, Chile y México, los países con más producción científica en América Latina ${ }^{6}$ y detalla el índice-h que generan en base a sus artículos citables publicados entre los años 2004 y 2006. Por de pronto, la mitad de los títulos registrados no están incorporados en las bases más amplias del ISI, i.e. Web of Science y tan sólo 3 (ver Tabla 1) están contenidos en el JCR en la categoría Medicine, Internal \& General. Los otros títulos detallados en la Tabla 2 y que registra el ISI están vinculados a otras categorías biomédicas.

Como se señala en la Tabla 2 la Revista Médica de Chile, con un índice-h =14 y 555 documentos citables en tres años se ubica en el cuarto y segundo lugar, respectivamente. Lidera el ranking el Brazilian Journal of Medical and Biological Research con un Factor de Impacto 2006 de 1.075 y que en el ISI está categorizado por la naturaleza principal de sus artículos, en dos ámbitos: i.Biology y ii.- Medicine, Research \& Experimental, claramente distinguible del rasgo que se le adscribe a la Revista Médica de Chile. Lo mismo acontece con la segunda revista, Archives of Medical Research que también está catalogada en Medicine, Research \& Experimental y que exhibe un Factor de Impacto 2006 de 1.272. El índice-h combina el efecto del tamaño del universo (número de artículos) con su distribución de citas en un período de tiempo determinado. Así, el tamaño de la muestra influye y se observan diferencias entre disciplinas $^{12}$. Por la forma de catalogar en la que se sustenta el ISI, las revistas Medicina-Buenos Aires y la Revista Médica de Chile serían más comparables entre sí. Como se observa en la Tabla 2 éstas generan un índice-h bastante similar y notoriamente más elevado al resto, exceptuando al Sao Paulo Medical Journal que no está incluido en el ISI.

Actualmente los programas del Web of Science también permiten inferir indicadores. Para la última 
Tabla 2. Indicadores de Revistas categorizadas en M edicina, M edicina M iscelánea de acuerdo a SC Imago*, 2004- 2006

\begin{tabular}{|c|c|c|c|c|c|}
\hline Revista* & ISI & $\begin{array}{c}\text { Índice } \\
\text {-h }\end{array}$ & $\begin{array}{c}\text { Citas } \\
\text { (3 años) }\end{array}$ & $\begin{array}{l}\text { Doc citables } \\
3 \text { años }\end{array}$ & País \\
\hline Brazilian Joumal of Medical and Biological Research & SI & 30 & 874 & 677 & Brasil \\
\hline Archives of Medical Research & SI & 24 & 492 & 272 & México \\
\hline Medicina - Buenos Aires & SI & 15 & 82 & 220 & Argentina \\
\hline Revista Médica de Chile & SI & 14 & 309 & 555 & Chile \\
\hline Sao Paulo Medical Journal & NO & 10 & 121 & 217 & Brasil \\
\hline Revista de Investigación Clínica & SI & 9 & 67 & 258 & México \\
\hline Genetics and Molecular Research & SI & 9 & 200 & 130 & Brasil \\
\hline Revista da Associacao Medica Brasileira & SI & 8 & 75 & 237 & Brasil \\
\hline Ann Hepatol & SI & 8 & 160 & 106 & México \\
\hline Brazilian Joumal of Biology & NO & 7 & 136 & 274 & Brasil \\
\hline Gaceta Médica de México & SI & 7 & 49 & 273 & México \\
\hline Acta Gastroenterológica Latinoamericana & SI & 7 & 15 & 73 & Argentina \\
\hline Revista de Nutricao & SI & 5 & 75 & 164 & Brasil \\
\hline Revista Brasileira de Otominolaringologia & NO & 4 & 43 & 399 & Brasil \\
\hline Revista Latino-Americano de Enfermagem & SI & 4 & 151 & 371 & Brasil \\
\hline Prensa Médica Argentina & SI & 4 & 1 & 369 & Argentina \\
\hline Revista Brasileira de Medicina & SI & 4 & 11 & 314 & Brasil \\
\hline Revista Alergia México & NO & 4 & 7 & 69 & México \\
\hline Clinics & SI & 4 & 83 & 52 & Brasil \\
\hline Acta Odontológica Latinoamericana: AOL & NO & 4 & 1 & 23 & Argentina \\
\hline Revista de la Facultad de Ciencias Médicas (Cóndoba) & NO & 3 & 6 & 43 & Argentina \\
\hline Revista Brasileira de Enfermagem & NO & 2 & 30 & 375 & Brasil \\
\hline Cirugía y Cirujanos & NO & 2 & 23 & 203 & México \\
\hline Revista da Escola de Enfermagem da U S P & SI & 2 & 15 & 164 & Brasil \\
\hline Vertex & NO & 2 & 10 & 156 & Argentina \\
\hline Acta Scientiarum - Biological Sciences & NO & 2 & 19 & 156 & Brasil \\
\hline Historia, Ciencias, Saude-Manguinhos & NO & 2 & 10 & 116 & Brasil \\
\hline PRO-FONO: Revista de Actualizacao Cientifica & NO & 2 & 2 & 89 & Brasil \\
\hline Revista Chilena de Infectología & SI & 2 & 16 & 49 & Chile \\
\hline Medicina - Universidad de Sao Paulo & NO & 2 & 4 & 19 & Brasil \\
\hline Acta Scientiarum - Health Sciences & NO & 1 & 5 & 106 & Brasil \\
\hline Revista Gaucha de Enfermagem/EENFUFRGS & NO & 1 & 13 & 105 & Brasil \\
\hline Acta Cirurgica Brasileira & NO & 1 & 11 & 69 & Brasil \\
\hline Revista Médica del Instituto Mexicano del Seguro Social & NO & 1 & 0 & 38 & México \\
\hline Salud (i) Sciencia & NO & 0 & 0 & 10 & Argentina \\
\hline Revista Brasileira de Otominolaringologia (English ed.) & NO & 0 & 0 & 3 & Brasil \\
\hline
\end{tabular}

* http://www.scimagojr.com. SCImago Research Group, Copyright 2007. Data Source: Scopus ${ }^{\circledR}$ ** Web of Science, Thomson ISI, 29 de abril 2008.

década (1998-2007) la Revista Médica de Chile registra 2.130 documentos publicados, con una cita promedio de 1,3 cada uno y un índice-h de 13. Este valor del índice-h es el mismo que alcanza la revista Medicina en la misma década a través de sus 1.448 documentos citados 1,16 veces en promedio. En ambos casos se considera el registro completo independientemente del tipo de artículo. De los 2.130 documentos publicados por la Revista Médica deChile, 1.693 están registrados como artículos, 188 son letters, 143 reviews, 67 material editorial, 23 ítems bibliográficos, 15 correcciones y uno está clasificado como bibliografía. 
Artículos más citados. De particular interés resulta observar la dinámica de crecimiento de las citas que acumulan anualmente los artículos publicados por la Revista Médica de Chile. La Figura 1 evidencia un incremento relevante cuyo rango supera $1.000 \%$ en el período estudiado. Como consecuencia de ello debe esperarse que se incremente con la misma dinámica el número de citas promedio que convoca cada artículo. El notable aumento de citas se vincula, por cierto a un esfuerzo editorial cada vez más profesional $^{13,14}$, a un reconocimiento internacional en constante aumento y al hecho que los artículos están indexados en los principales registros y los artículos están disponibles en línea en www.scielo.cl. Sin embargo, es necesario vencer una conducta perjudicial que impregna a los países en vías de desarrollo, cuyos autores suelen omitir sin justificación alguna las referencias a trabajos de connacionales ${ }^{15}$. Con todo, el incremento de la visibilidad de la Revista y de la calidad de sus artículos se infiere, asimismo, al identificar los "Top Ten" en cuanto a citas acumuladas entre 1998 y 2007. En efecto, la Tabla 3 contiene los artículos publicados durante la última década, obteniendo el primero 38 citas y el último 16. Esto se compara muy favorablemente con el rendimiento de los diez primeros artículos en cuanto a acumulación de citas en los 15 años previos (1981-1995) ${ }^{2}$ donde el más alto obtuvo 27 referencias y el décimo de la lista acumuló 11 citas, a pesar que el tiempo acumulado en el período superaba en $50 \%$ a la ventana que se describe en la Tabla 3.

Es también notorio que durante la última década (1998-2007) el 50,56\% de los documentos publicados (ítems) por la Revista Médica de Chile fueron citados a mayo de 2008. Ello se compara muy favorablemente con $37,8 \%$ de los artículos publicados entre 1981 y 19952. Particularmente, porque el universo de los documentos considerados en el presente estudio, a diferencia del realizado para los años 1981-1995, incluyen ítems que provocan escasas citas (ver Materiales y Métodos).

De los 2.130 documentos publicados en la Revista Médica de Chile durante la década poco más de $40 \%$ están asociados a la Universidad de Chile. Como se observa en la Tabla 4, en lo que concierne a la filiación de los autores de los trabajos le sigue la Pontificia Universidad Católica de Chile con algo más que $25 \%$ y en tercer lugar, la Universidad de la Frontera con una proporción bastante más baja. Las siguen las demás instituciones, con el número de documentos aportados en el período de análisis. Es necesario tener en cuenta que el ámbito de la biomedicina genera en los bancos de datos un abanico de subáreas amplio y que la información contenida en la Tabla 4 sólo incluye Medicina General e Interna. También ocurre que artículos de otras revistas pueden ser clasificados por el ISI en más de una categoría.

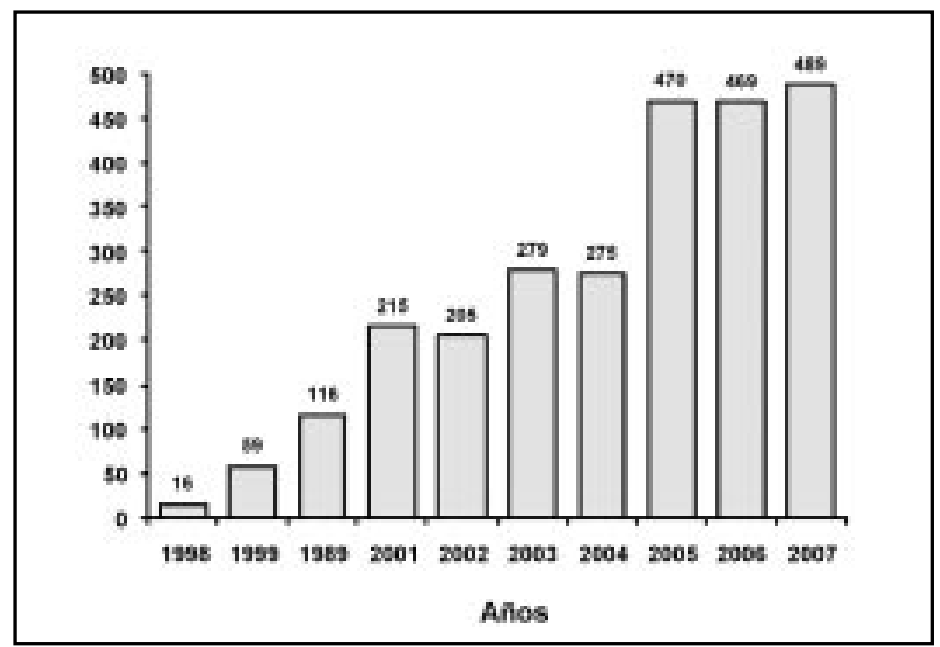

Figura 1. Citas anuales a artículos de la Revista Médica de Chile publicados entre 1998-2007¹.

${ }^{1}$ Web of Science 16 de abril de 2008. 


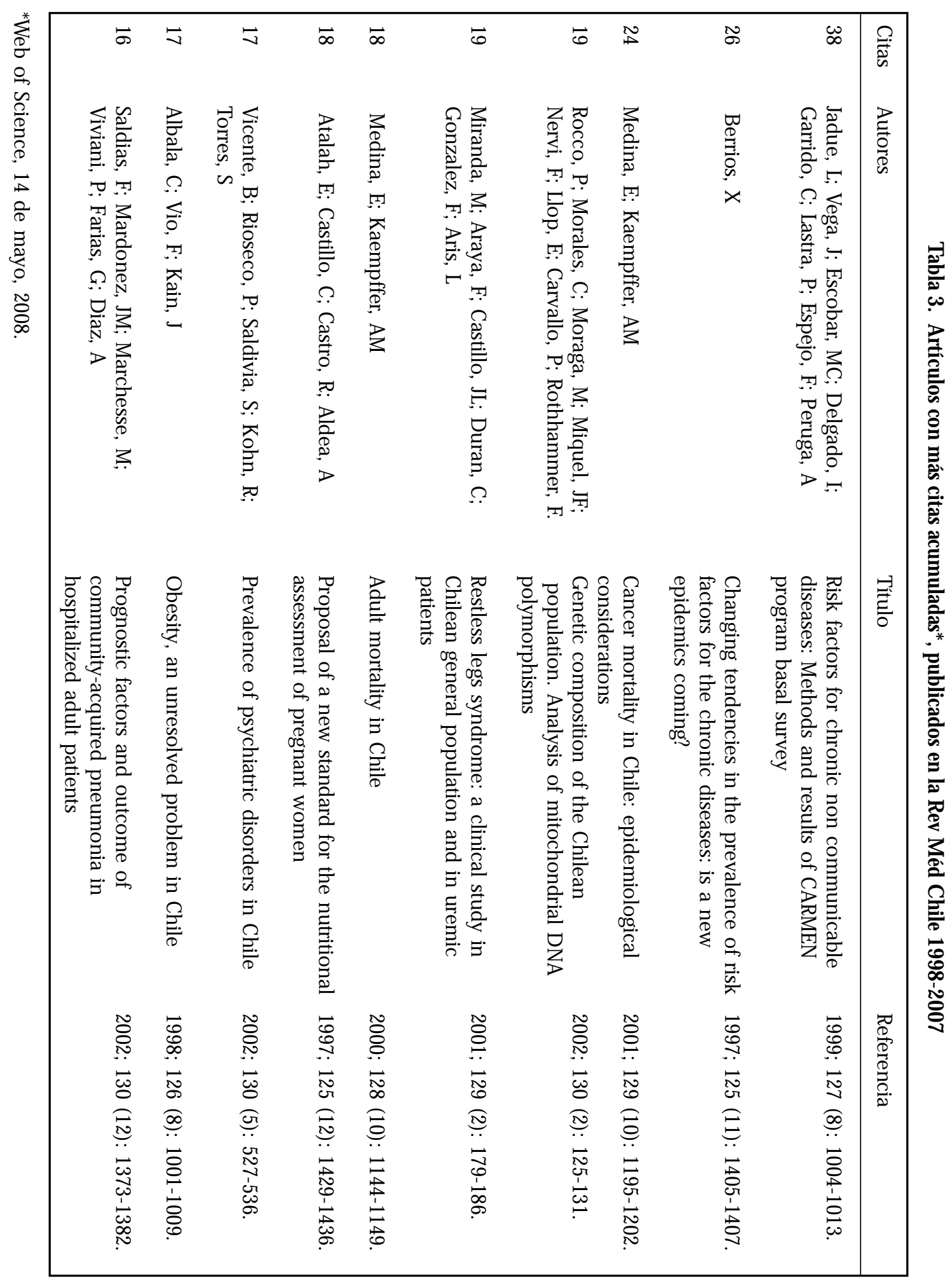


Tabla 4. N úmero de artículos* publicados en la Revista M édica de Chile por las instituciones que se indican (1998-2007)

\begin{tabular}{|lcr|}
\hline Institución & $\mathrm{N}^{\circ}$ artículos & $\%$ \\
\hline Universidad de Chile & 872 & 40,94 \\
P. Universidad Católica de Chile & 570 & 26,76 \\
Universidad de la Frontera & 94 & 4,41 \\
Universidad de Valparaíso & 74 & 3,47 \\
Universidad de Concepción & 67 & 3,15 \\
Hospital San Borja & 53 & 2,49 \\
Hospital del Salvador & 47 & 2,21 \\
Universidad Austral de Chile & 43 & 2,02 \\
Clínica Las Condes & 40 & 1,88 \\
Universidad de Los Andes & 36 & 1,69 \\
Hospital Sótero del Río & 36 & 1,69 \\
\hline
\end{tabular}

Se incluyen todos los ítems.

Fuente: Web of Science.

Dinámica de la investigación en Medicina en los países con más actividad en América Latina. Entre 1996 y el 2001, Argentina, Brasil, Chile y México no exhibiemon variaciones profundas en lo que concierne a publicaciones en Medicina. Empero, mientras el resto de los países mantuvo su nivel en el siguiente quinquenio, Brasil hace ostensible un salto cuantitativo muy grande. Como se observa en la Figura 2, consistente con una política pública nacional que apunta a jugar un rol protagónico en la sociedad del conocimiento donde las necesidades de investigación para la educación superior, la innovación y la apropiación social de la ciencia son una exigencia insoslayable, Brasil consolida su liderazgo en la región. De hecho, ya en el año 2003 Brasil produjo 1,45\% de los artículos en C \& T

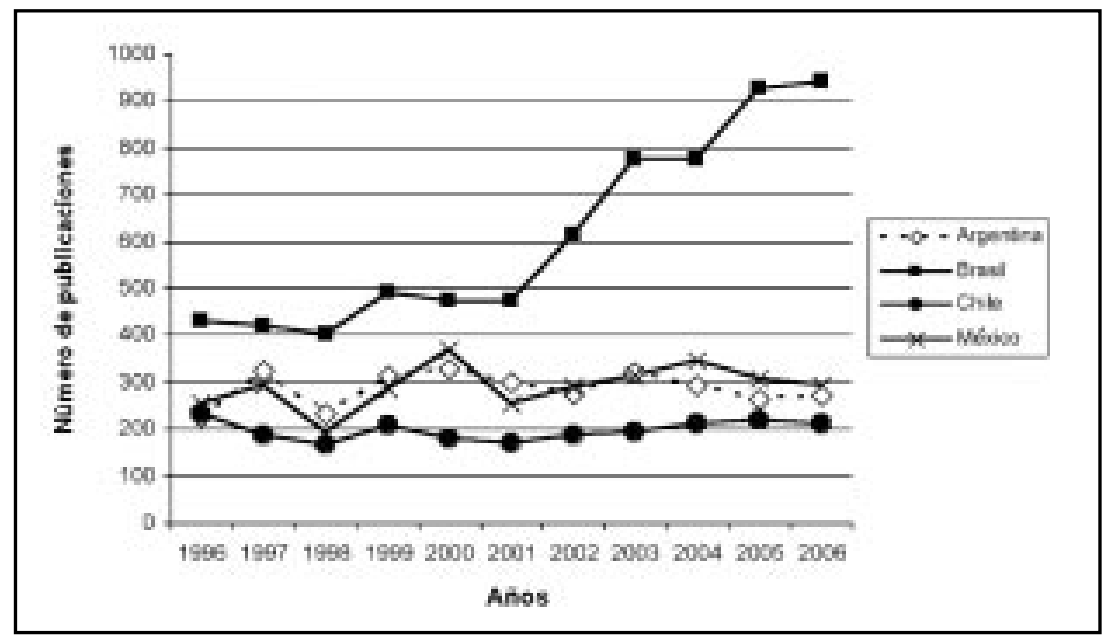

Figura 2. Número de artículos (documentos citables) registrados en el área de Medicina y categoría Medicina miscelánea ${ }^{1}$.

${ }^{1}$ SCImago Research Group, Copyright 2007. Data Source: Scopus ${ }^{\circledR}$. 
publicados en revistas de corriente principal y al año 2004 el 78\% de los Ph.D,s graduados en programas acreditados en América Latina ${ }^{6}$. Como se observa en la Figura 3 la calidad de lo publicado por Brasil en el ámbito de la Medicina es claramente distinguible cuando se identifica el índice-h. Chile se posiciona en un cuarto lugar en esta categoría.
A la luz de los indicadores comparativos, la contribución realizada por la Revista Médica de Chile se hace aún más relevante toda vez que en la categoría Medicina General e Interna, tiene un aporte de proporciones. Por ello es de enorme importancia el significativo incremento de su visibilidad e impacto, que se revela a través de los indicadores que aquí se presentan.

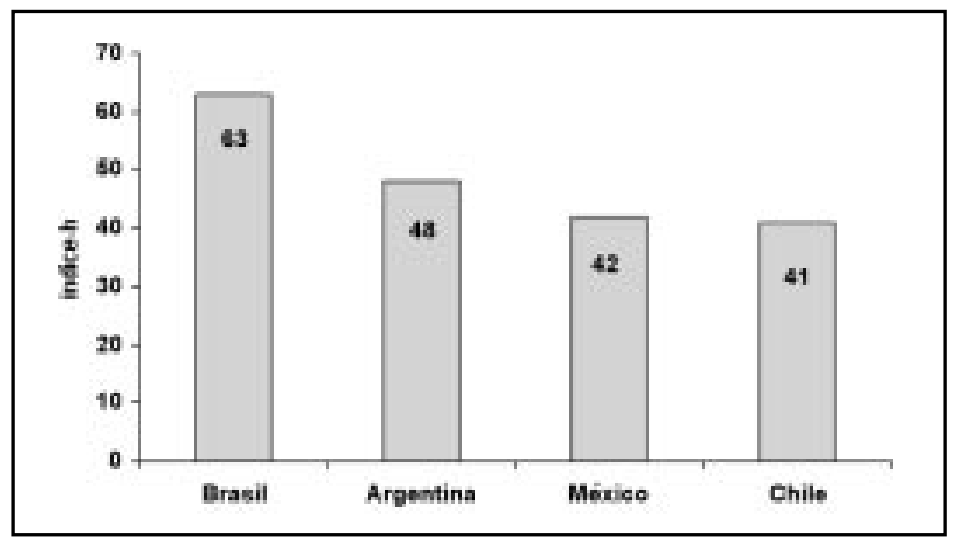

Figura 3. Indice-h de los documentos citables publicados por cada país (1996-2006) ${ }^{1}$

${ }^{1}$ SCImago Research Group, Copyright 2007. Data Source: Scopus ${ }^{\circledR}$

\section{REFERENCIAS}

1. KRAUSKopf M. Epistemometría, a term contributing to express the meaning and potential methodologies of scientometrics in Spanish speaking countries. Scientometrics 1994; 30: 425-8.

2. KRAUSKOPF M. Una radiografía epistemométrica de la Revista Médica de Chile. Rev Méd Chile 1997; 125: 775-80.

3. SANTelices B, Contreras C, Morata D. Inversión nacional en investigación y desamollo 1995-2004, en Análisis y proyecciones de la Ciencia Chilena 2005 (eds. Jorge E Allende, Jorge Babul, Servet Martínez, Tito Ureta) Academia Chilena de Ciencias pp 109-132.

4. SCIMmago (2007) SJR-SCImago Journal \& Country Rank. Retrieved April 23, 2008 from http: // www.scimagojr.com

5. OECD Reviews on Innovation Policy in Chile, Latin American Economic Outlook 2008, OECD, Ministerio de Economía y Ministerio de Hacienda, Gobierno de Chile, CD 2007.

6. Hermes-Lima M, Santos NCF, Aiencastro ACR, Ferreira ST. Whither Latin America? Trends and Challenges of Science in Latin America IUBMB Life 2007; 59: 199-210.
7. HiRsCH JE. An index to quantify an individual's scientific output. Proc Nat Acad Sci (USA) 2005; 102: 16569-72.

8. Thomson ISI Web of Science and Journal of Citation Report.

9. SCImago Research Group, Copyright 2007. Data Source: Scopus ${ }^{\circledR}$.

10. Collazo-Reyes F, Luna-Morales ME, Russell JM, Perez-Angon M. Publication and citation patterns of Latin American \& Caribbean journals in the SCI and SSCI from 1995 to 2004. Scientometrics 2008; 75: $145-61$.

11. http: //www.info.scopus.com/

12. Csajbók E, Berhidi A, Vasas L, Schubert A. Hirschindex for countries based on Essential Science Indicators data. Scientometrics 2007; 73: 91-117.

13. Reyes H. El Aniversario 135 de la Revista Médica de Chile. Rev Méd Chile 2007; 135: 7-10.

14. Lee RV. Medical journals: Old, not senescent. A tribute to Revista Médica de Chile on its $135^{\text {th }}$ Anniversary. Rev Méd Chile 2007; 135: 823-8.

15. Rioseco A. Citas de autores nacionales en la Revista Médica de Chile. Rev Méd Chile 2004; 132: 645. 